\title{
Tumor Accumulation of Radiolabeled Bevacizumab due to Targeting of Cell- and Matrix-Associated VEGF-A Isoforms
}

\author{
Thamar H. Stollman, ${ }^{1}$ Marian G.W. Scheer, ${ }^{1}$ Gerben M. Franssen, ${ }^{2}$ Kiek N. Verrijp, ${ }^{3}$ Wim J.G. Oyen, ${ }^{2}$ \\ Theo J.M. Ruers, ${ }^{1}$ William P.J. Leenders, ${ }^{3}$ and Otto C. Boerman ${ }^{2}$
}

\begin{abstract}
Purpose: Vascular endothelial growth factor-A (VEGF-A) is one of the most important factors inducing angiogenesis in tumors. Nine splice-variant isoforms of VEGF-A have been identified, each having different properties. Recently, we showed that radiolabeled anti-VEGF monoclonal antibody, bevacizumab, accumulates specifically in VEGF-A expressing tumors. In this study, we investigated in a nude mouse model which VEGF-isoforms are responsible for tumor accretion.

Materials and Methods: The humanized anti-VEGF-A antibody, A.4.6.1. (bevacizumab), was radiolabeled with In-111. The originally VEGF-negative Mel57 tumor was transfected with different VEGF isoforms (VEGF-121, VEGF-165, and VEGF-189). The obtained melanoma xenografts specifically expressing different VEGF-isoforms were used in mice. The bevacizumab uptake was examined in biodistribution studies and by gamma-camera imaging.

Results: The tumor cell line expressing VEGF-121 did not show specific uptake, most likely as a result of the fact that this isoform is freely diffusible. Tumors expressing VEGF-165 and -189 were clearly visualized by using gamma-camera imaging.

Conclusion: The accumulation of radiolabeled bevacizumab in the tumor is due to interaction with VEGF-A isoforms that are associated with the tumor cell surface and/or the extracellular matrix. Scintigraphic imaging of the expression of these VEGF isoforms may thus be useful to predict response to angiogenic therapy.
\end{abstract}

Key words: VEGF-A, isoforms, angiogenesis, bevacizumab, scintigraphy, Avastin

\section{Introduction}

$\mathbf{T}$ umors cannot grow beyond a size of $1-2 \mathrm{~mm}^{3}$ unless new blood vessels are formed for oxygen and nutrient supply. ${ }^{1}$ Angiogenesis is a complex process that is regulated by the actions of prol and antiangiogenic cytokines and hormones. Vascular endothelial growth factor-A (VEGF-A) is one of the most important proangiogenic growth factors. A wellcharacterized activity of VEGF-A is its ability to promote the growth and migration of vascular endothelial cells derived from arteries, veins, and even lymphatics. ${ }^{2}$ VEGF-A is the best-characterized member of the VEGF family and is thought to be the predominant and most critical regulator of the development of the vascular system in various tumors. ${ }^{3}$

The human VEGF-A gene is organized in nine exons, separated by eight introns ${ }^{4}$ and is localized on chromosome 6p21.3. ${ }^{5}$ Alternative splicing may result in the generation of six isoforms, having 121, 145, 165, 183, 189, and 206 amino acids, respectively, after signal-sequence cleavage. The amino acids encoded by exons $1-5$ and 8 are contained in all isoforms. Exons 6 and 7 encode heparin-binding domains, which influence receptor binding and solubility. VEGF-189 and -206 contain a domain encoded by exon 6 and bind to heparin and heparan sulphate proteoglycans (HSPGs) with high affinity and are almost completely sequestered in the extracellular matrix (ECM). VEGF-165 is the most predominant isoform ${ }^{6}$ : It contains the exon 7-encoded domain, but lacks exon 6 and has intermediate properties; it is secreted, but a significant portion is bound to HSPGs on the cell surface and ECM. $^{7}$ VEGF-121, which lacks both exons 6 and 7, does not bind to heparin and HSPGs and is, consequently, freely diffusible. ${ }^{8}$ We previously demonstrated, in a brain metastases

Departments of ${ }^{1}$ Surgery, ${ }^{2}$ Nuclear Medicine, and ${ }^{3}$ Pathology, Radboud University Nijmegen Medical Center, Nijmegen, The Netherlands.

Address correspondence to: Thamar H. Stollman; Department of Surgery, Radboud University Nijmegen Medical Center; P.O. Box 9101, 6500 HB Nijmegen, The Netherlands; Tel.: +3124-3611340

E-mail: T.Stollman@chir.umcn.nl 
model, that VEGF-165 and -189 are angiogenic, whereas VEGF-121 mainly induced vasodilatation and vascular permeability. In clinical tumors, the 121 and 165 isoforms, and to a lesser extent, VEGF-A189, are the predominant VEGF isoforms. ${ }^{9,10}$

Bevacizumab is a humanized variant of the anti-VEGF-A monoclonal antibody $(\mathrm{mAb})$, A.4.6.1. It is directed aginst a common epitope encoded by exon 4 and expressed on all VEGF-A isoforms, preventing interaction with the tyrosine kinase receptors, VEGFR-1 and $-2 .{ }^{11}$ It has been shown that antiangiogenic properties of bevacizumab result in growth inhibition of human tumors in nude mice. ${ }^{12}$ Bevacizumab was the first Food and Drug Administration-approved clinical agent to target tumor angiogenesis and has been registered in February 2004 for the first-line treatment of metastatic colorectal cancer in combination with Fluorouracel (5-FU)-based chemotherapy. ${ }^{13}$ In a recent trial, the addition of bevacizumab to standard chemotherapy also produced a significant survival benefit in patients with advanced metastatic non-smallcell lung cancer, ${ }^{14}$ and it was approved for initial treatment of irresectable non-small-cell lung cancer in combination with carboplatin and paclitaxel in October 2006.

The marked variation in functional anatomy and pathophysiology within human tumors and within individual patients may account for the high variability of responses observed in patients. ${ }^{15}$ For example, in vessel-dense tissues such as the liver, tumors may profit from preexistent vasculature, thereby reducing the dependency on angiogenesis. Liver metastases from colorectal adenocarcinomas grow in three patterns with different angiogenesis and desmoplasia, as pointed out by Vermeulen et al. ${ }^{16}$ As a result, there is an urgent need for reliable markers that can predict which patients are most likely to respond to anti-VEGF therapy.

In a previous study, we showed that VEGF-A expression by the LS174T colon tumor xenografts could be visualized by gamma-camera imaging after the intravenous (i.v.) injection of In-111-labeled anti-VEGF mAb. ${ }^{17,18}$ VEGF-A expression in these xenografts was limited to the 121 and 165 amino-acid variants. However, it remains unclear which isoform(s) were visualized in these imaging experiments. The aim of this study was to determine which VEGF isoforms were actually targeted. Therefore, we analyzed bevacizumab uptake in melanoma xenografts in nude mice selectively expressing VEGF-121, -165 , or -189 .

\section{Materials and Methods}

\section{Tumor model}

All animal experiments were approved by the Animal Experiments Committee of the Radboud University Nijmegen Medical Center (Nijmegen, The Netherlands) and performed in accordance with their guidelines. Male nude BALB/c mice, 6-8 weeks old, were used after an acclimatization period of 2 weeks. The mice were housed (4 per cage) in a controlled environment with light-dark cycle conditions (12 hour light and $12 \mathrm{~h}$ dark) and had free access to water and food. Stable transfectants of the Mel57 human melanoma cell line expressing VEGF-121, -165, or -189 were obtained, as described previously. ${ }^{19}$ Expression of proteins of the appropriate size in conditioned medium was confirmed by using Western blot, using anti-VEGF antibody A20 (Santa Cruz Biotechnology, Santa Cruz, CA).

\section{Radiolabeling and quality control}

For labeling with In-111, bevacizumab was conjugated with isothiocyanato-benzyl-DTPA (diethylene triamine penteacetic acid), as described previously. ${ }^{20}$ Bevacizumab-DTPA $(50 \mu \mathrm{g})$ was incubated with $20 \mathrm{MBq}$ of In-111 in $0.15 \mathrm{M}$ of ammonium acetate buffer ( $\mathrm{pH} 5.5$ ) for 30 minutes. The radiolabeled antibody preparations were purified by gel filtration on a PD-10 column (Amersham Biosciences, Roosendaal, The Netherlands) eluted with phosphate buffered saline (PBS) in $0.5 \%$ bovine serum calbumin (BSA). The radiochemical purity $(\mathrm{RCP})$ of the radiolabeled $\mathrm{mAb}$ (specific activity $0.3 \mathrm{MBq} / \mu \mathrm{g}$ ) was determined by instant thin-layer chromatography (ITLC) and exceeded $97 \%$ for all preparations used in the studies.

\section{VEGF-A levels in tumor xenografts}

From the frozen melanoma xenografts expressing the different VEGF isoforms (VEGF-121, -165, and -189), thick cryosections were cut and pooled in RIPA buffer, $(150 \mathrm{mM}$ of $\mathrm{NaCl}, 10 \mathrm{mM}$ of Tris- $\mathrm{HCl}, \mathrm{pH} 7.4,1 \mathrm{mM}$ of ethylene diamine tetraacetic acid, $1 \% \mathrm{NP}-40$, and $0.5 \%$ sodiumdeoxycholate) and a protease inhibitors cocktail (Roche, Basel, Switzerland) containing heparin $(5 \mathrm{U} / \mathrm{mL})$ to release matrix-bound isoforms of VEGF-A. Sections were homogenized, incubated on ice for 20 minutes, and centrifuged $(800 \mathrm{~g}$, for 20 minutes at $4^{\circ} \mathrm{C}$ ) to prepare a clear lysate. Protein concentrations were determined by the Bradford method (Biorad, Hercules, CA), and VEGF-A concentrations were measured by enzyme linked immunosorbent assay (ELISA) as described previously. ${ }^{21}$ This ELISA detects all VEGF-isoforms.

\section{Biodistribution and imaging experiments}

Nude mice were injected subcutaneously (s.c.) in the left flank with $0.2 \mathrm{~mL}$ of Matrige ${ }^{\circledR}$ (Becton Dickinson, Franklin Lakes, NJ) containing $4 \times 10^{5}$ Mel57-wt, Mel57-VEGF-121, Mel57-VEGF-165, or Mel57-VEGF-189 cells $(n=8$ for each group). Approximately 14 days later, mice bearing 2-3 mmdiameter tumors (approximately $0.1 \mathrm{~g}$ ) received an i.v. injection (via the tail vein) of $0.2 \mathrm{MBq}$ of In-111-bevacizumab $(0.2 \mathrm{~mL})$. Three (3) mice from each group were coinjected with an excess of unlabeled bevacizumab (300 $\mu \mathrm{g})$. Three (3) days after injection, mice were killed by $\mathrm{CO}_{2} / \mathrm{O}_{2}$ asphyxiation and tissues (blood, muscle, lung, spleen, kidney, liver, and small intestine) were dissected and weighed. The radioactivity in the tissues was measured in a gamma-counter (Wizard; Pharmacia-LKB Uppsala, Sweden). Injection standards were counted simultaneously for correction of decay. The activity concentration in the samples was calculated and expressed as percent injected dose $(\% \mathrm{ID} / \mathrm{g})$ of tissue.

\section{Scintigraphic imaging}

For imaging experiments, mice with 3-6 mm-diameter s.c. Mel57 tumors expressing the different isoforms $(n=3$ in each group) were i.v. injected with $1 \mathrm{MBq}$ of In-111-bevacizumab (in $0.2 \mathrm{~mL}$ ). Scintigraphic images were acquired immediately and at 1,3 , and 7 days postinjection. For imaging, mice were anesthetized by isoflurane $/ \mathrm{N}_{2} \mathrm{O} / \mathrm{O}_{2}$ inhalation anesthesia and placed prone on a single-head gamma camera (Orbitor; Siemens, Hoffman Estates, IL) equipped with a parallel-hole medium-energy collimator. Images (100,000 counts/image) 
were stored digitally in a $256 \times 256$ matrix. After the acquisition of the last images, mice were killed and tumors were snap-frozen for further analysis.

\section{Results}

\section{VEGF-A levels in tumor xenografts}

VEGF-A levels in tumor tissue extract were determined by using an ELISA assay. VEGF levels in the tumor extracts varied from $0.24 \mu \mathrm{g} / \mathrm{g}$ per tumor in the Mel57-wt xenograft to $4.03 \mu \mathrm{g} / \mathrm{g}$ per tumor in the Mel57-VEGF-165 xenograft. The VEGF levels in the Mel57-VEGF-121 and Mel57-VEGF189 xenograft amounted to 0.64 and $1.13 \mu \mathrm{g} / \mathrm{g}$ per tumor, respectively. We found no correlation between the VEGF levels in the tumor xenografts and the specific accumulation of radiolabeled bevacizumab (Spearman correlation, 0.8; $p<0.20$ ).

\section{Biodistribution experiment}

Expression of VEGF-A isoforms by the respective Mel57 transfectants is shown in Figure 1. The different transfectants exclusively produce the transfected isoform. The biodistribution of In-111-bevacizumab in mice with s.c. Mel57 tumors expressing different VEGF-A isoforms is summarized in Figure 2A-2D. Mel57 wild-type xenografts, known to express low levels of VEGF-A, ${ }^{22}$ showed an In-111-bevacizumab concentration of $8.6 \pm 1.2 \% \mathrm{ID} / \mathrm{g}$. In the presence of an excess of unlabeled bevacizumab, the tumor uptake of the In-111-labeled antibody was reduced significantly: $3.9 \pm 0.34 \% \mathrm{ID} / \mathrm{g}$, indicating that half of the antibody uptake was VEGF mediated. The concentration of In-111-bevacizumab in the Mel57-VEGF121 tumor was $20 \pm 3 \% \mathrm{ID} / \mathrm{g}$. Uptake in the Mel57-VEGF-121

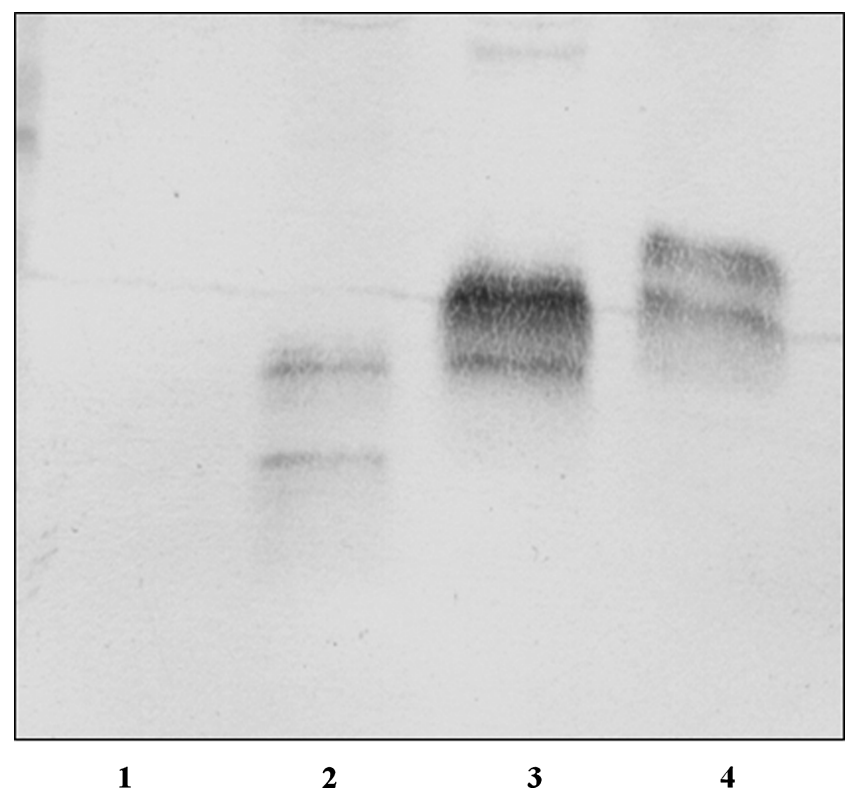

FIG. 1. Expression of vascular endothelial growth factor (VEGF) isoforms by Mel57 transfectants in vitro. A Western blot is shown containing conditioned medium of Mel57 (lane 1), Mel57-VEGF-A121 (lane 2), Mel57-VEGF-A165 (lane 3), and Mel57-VEGF-A189 (lane 4). Note the absence of VEGF in parental Mel57 cells. Every isoform is secreted as a glycosylated protein, resulting in the doublet character. tumors and normal tissues was not reduced in the presence of $300 \mu \mathrm{g}$ of unlabeled antibody, indicating that In-111bevacizumab accumulated only nonspecifically in these tumors. The concentration of In-111-bevacizumab in the Mel57-VEGF-189 tumor was $37 \pm 13 \%$ ID/g. Mel57-VEGF165 tumors demonstrated an extremely high bevacizumab uptake of $64 \pm 12 \% \mathrm{ID} / \mathrm{g}$, resulting in a tumor-to-blood ratio as high as 7.9 at 3 days postinjection. The concentration of In-111-bevacizumab in the Mel57-VEGF-165 and Mel57VEGF-189 tumors decreased significantly upon the coinjection of an excess unlabeled antibody ( 20 and $4 \% \mathrm{ID} / \mathrm{g}$ respectively), indicating that the major part of bevacizumab uptake in these tumors was specific (VEGF mediated).

\section{Scintigraphic imaging}

The images acquired at several time points after injection are shown in Figure 3. Immediately after injection (day 0), radioactivity was mainly present in the heart region and wellperfused organs. At day 3 post injection, activity had accumulated in the VEGF-165- and -189-positive tumors and had cleared from the circulation and nontarget organs, resulting in the distinct visualization of these tumors. Image quality further improved at day 7. The Mel57 wild-type tumors and the Mel57 tumors expressing VEGF-121 were not delineated in the images acquired 3 and 7 days postinjection, confirming the lack of specific accumulation of the antibody in these tumors.

\section{Discussion}

We and others recently demonstrated that radiolabeled bevacizumab specifically accumulates in VEGF-A expressing tumors xenografted in nude mice. ${ }^{17,23}$ The In-111-labeled antibody allowed the detection of VEGF-A expression noninvasively by scintigraphic imaging. Bevacizumab is reported to have a high affinity for all VEGF-A isoforms, but it was unclear which isoforms expressed in the tumor were actually targeted and caused the specific accumulation in the tumor. Because previous work has shown that the larger isoforms of VEGF-A are more potent inducers of angiogenesis, ${ }^{9}$ it is important to know which VEGF-A isoform(s) are visualized during anti-VEGF imaging.

To determine which of the isoforms are responsible for the specific bevacizumab uptake, we used stable transfectants of the human melanoma cell line, Mel57, expressing each isoform in vitro at levels of $30-100 \mathrm{ng} / \mathrm{mL}$ per $10^{6}$ cells in 48 hours. ${ }^{9}$ The parental Mel57 cell line produces minimal amounts of VEGF-A in vitro. ${ }^{22}$ Consequently, Mel57 xenografts in nude mice grow to necrotic tumors, whereas VEGF-A-165 expression by these tumors results in a highly vascularized phenotype. ${ }^{24}$ In the present study, we demonstrate that only expression of the cell- or matrix-associated VEGF-A isoforms (VEGF-165 and -189) resulted in the tumor accumulation of radiolabeled bevacizumab.

The low levels of VEGF-A produced by the parental Mel57 xenografts were reflected in a very low specific uptake of bevacizumab. Specificity was assessed by the coinjection of an excess of unlabeled antibody. Highest tumor uptake was observed in the Mel57 tumor expressing VEGF-A-165 (64 \pm 12 $\%$ ID/g). The Mel57-VEGF-189 tumor also showed a high and specific tumor uptake. This high antibody uptake most likely is due to the properties of VEGF-165 and -189: A significant portion remains bound to the cell surface and the extracellular 

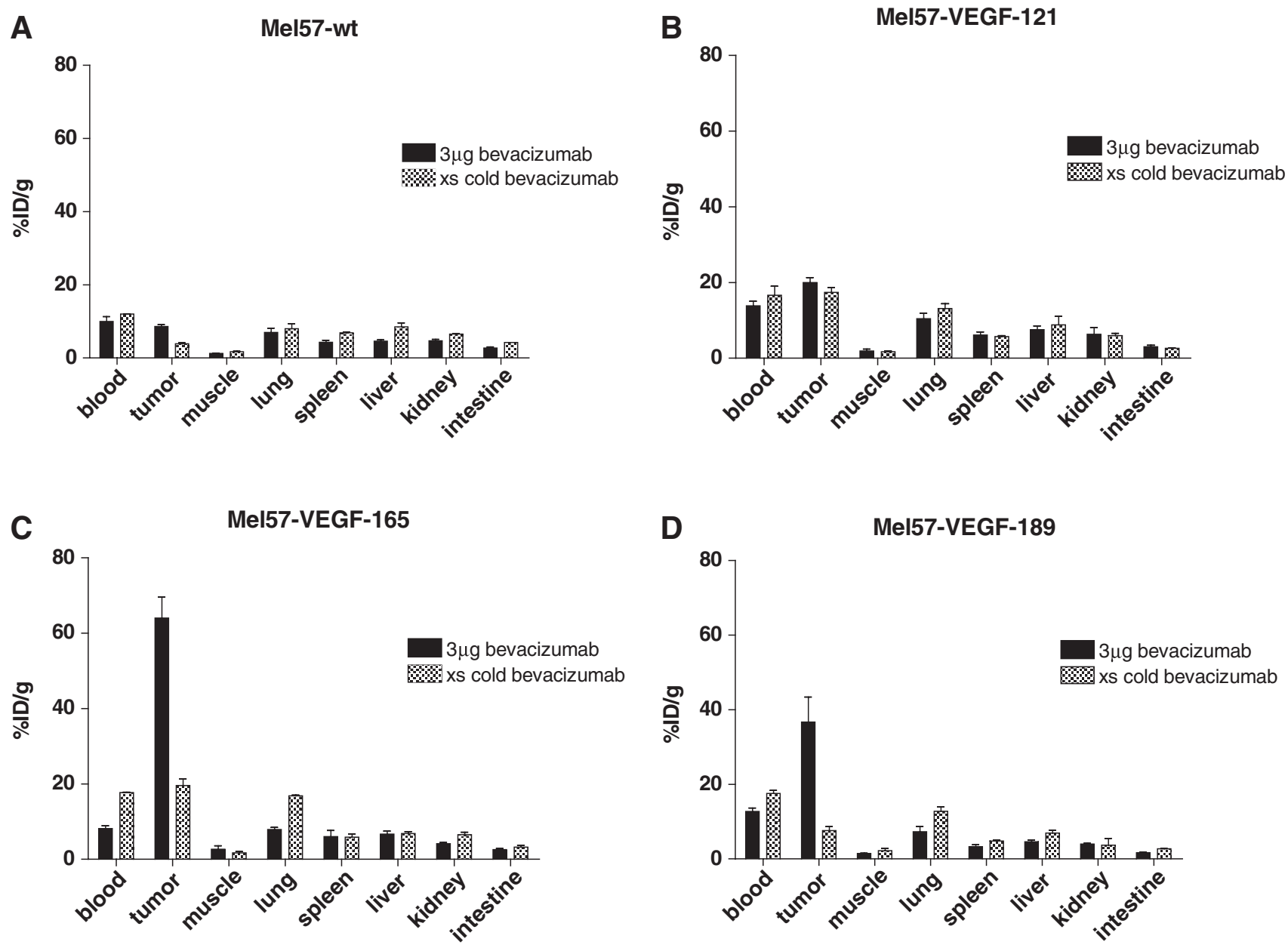

FIG. 2. Biodistribution of In-111-bevacizumab in athymic male mice with subcutaneous Mel57 tumors at 3 days postinjection (8 mice per group, with 3 of each group coinjected with an excess of $300 \mu \mathrm{g}$ of unlabeled bevacizumab) (A) Mel57-wt; (B) Mel57-VEGF-121; (C) Mel57-VEGF-165; and (D) Mel57-VEGF-189. VEGF, vascular endothelial growth factor.

matrix. Interestingly, uptake in the Mel57-VEGF-121 tumors was not reduced when an excess of unlabeled antibody was coinjected, indicating that the antibody localized in these tumors nonspecifically. This can be explained by the fact that VEGF-121 is not retained in these tumors, resulting in the formation of soluble VEGF-antibody immune complexes that are not retained in the tumor.

In this study, major differences in nonspecific uptake of bevacizumab in the Mel57 variants were observed. The concentration of radiolabeled bevacizumab, when coinjecting an excess dose of unlabeled antibody, was relatively high in the tumors expressing the VEGF-isoforms, 121 and 165: $17.4 \pm 2.23$ and $19.6 \pm 3.01 \% \mathrm{ID} / \mathrm{g}$, respectively. This may be the result of differences in blood volume, vascular permeability, and/or interstitial fluid pressure in the tumor xenografts, as VEGF-121 is reported to induce vasodilatation and to enhance vascular permeability. When grown in a brain metastasis model, Mel57 tumors that exclusively express VEGF-121 are characterized by a relative absence of intratumoral blood vessels, whereas highly dilated and hyperpermeable supposedly preexistent vessels are present in a peritumoral rim. VEGF-165 and -189 induce the formation of an intratumoral neovascular bed, consisting of irregularly dilated and leaky vessels. This is more prominent in VEGF- 165-expressing tumors, when compared with VEGF-189 tumors. ${ }^{9}$ Thus, the enhanced nonspecific localization in the VEGF-121 and -165 tumors may be due to enhanced vascular permeability, which may cause enhanced extravasation of bevacizumab in this tumor. However, further studies are needed to make proof of this assumption. Such an experiment should measure the vascular permeability of the tumor xenograft transfected with VEGF-121.

The localization of radiolabeled bevacizumab in mice bearing s.c. xenografted human tumors expressing particular VEGF isoforms was also studied by scintigraphic imaging. The tumors expressing VEGF-165 and -189 were clearly visualized, whereas the tumors expressing the VEGF-121 isoform could not be delineated. VEGF-165 and -189 are extracellular matrix-bound proteins and are known to be most capable of inducing angiogenesis. Imaging these VEGF isoforms may, therefore, be useful in selecting patients who are likely to respond to antiangiogenic therapy.

However, part of the response to antiangiogenic therapy could be due to reducing the interstitial fluid pressure in the tumor. VEGF-121 may not be the main isoform responsible for angiogenesis, but this isoform is well capable of inducing vasodilatation and enhancing vascular permeability. This results in inefficient blood flow and high interstitial fluid 

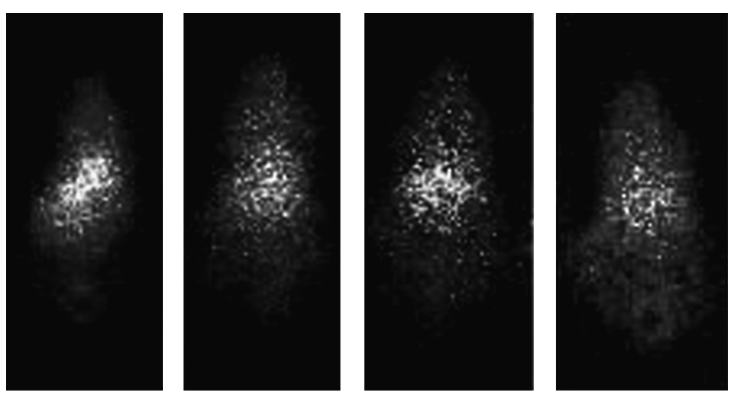

Mel57-wt
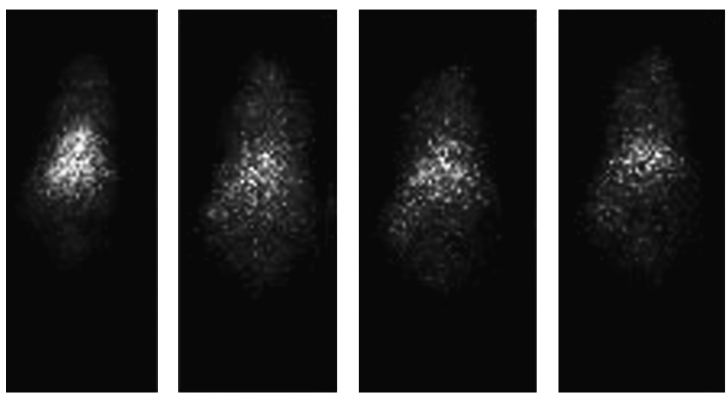

Mel57-VEGF-121
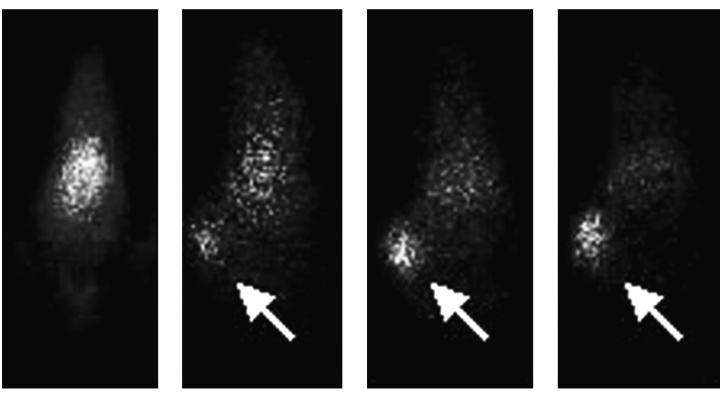

Mel57-VEGF-165

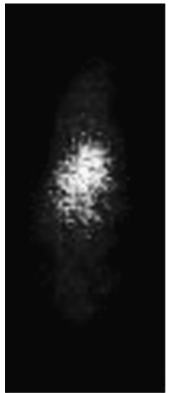

0

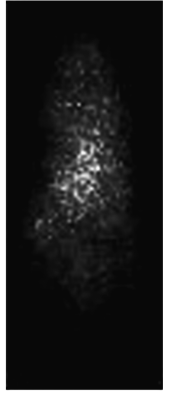

1

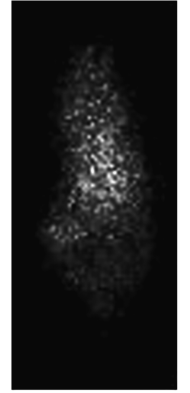

3

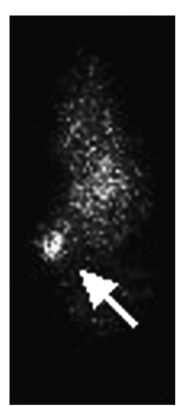

7 days

Mel57-VEGF-189

FIG. 3. Scintigraphic images of athymic male mice with subcutaneous Mel57 tumors expressing different VEGF isoforms immediately after injection and at 1, 3, and 7 days postinjection of In-111-bevacizumab (1 MBq/mouse, $3 \mu \mathrm{g} / \mathrm{mouse})$. VEGF, vascular endothelial growth factor.

pressure in the tumor, which leads to decreased uptake of drugs or other therapeutics in tumors. Combining antiVEGF-therapy with chemotherapy has resulted in an antitumor effect, presumably by the normalization of the tumor vasculature, partly as a result of blocking the effect of VEGF-121. As described above, the tumors expressing the VEGF-121 isoform could not be delineated.

\section{Conclusions}

This study is the first to demonstrate the specific tumor localization of radiolabeled bevacizumab in xenografted human tumors expressing particular VEGF isoforms. Tu- mors expressing only VEGF-121 showed no specific uptake of radiolabeled bevacizumab, most likely as a result of the fact that this isoform is freely diffusible and is not retained in the tumor. Mel57-VEGF-165 and Mel57-VEGF-189 demonstrated relatively high, specific tumor uptake of the labeled antibody. Thus, scintigraphy with radiolabeled bevacizumab appears to allow in vivo visualization of the VEGF-A isoforms that are most capable of inducing angiogenesis and may thus be useful to predict response to antiangiogenic therapy. However, it should be noted that tumors with predominantly VEGF-121 expression will not be visualized, whereas such tumors might still be susceptible to avastininduced vessel normalization. 


\section{Disclosure Statement}

No competing financial conflicts exist.

\section{References}

1. Ferrara N. Vascular endothelial growth factor and the regulation of angiogenesis. Recent Prog Horm Res 2000;55: 15.

2. Ferrara N. Vascular endothelial growth factor: Basic science and clinical progress. Endocr Rev 2004;25:581.

3. Ferrara N, Carver-Moore K, Chen H, et al. Heterozygous embryonic lethality induced by targeted inactivation of the VEGF gene. Nature 1996;380:439.

4. Tischer E, Mitchell R, Hartman T, et al. The human gene for vascular endothelial growth factor. Multiple protein forms are encoded through alternative exon splicing. J Biol Chem 1991;266:11947.

5. Vincenti V, Cassano C, Rocchi M, et al. Assignment of the vascular endothelial growth factor gene to human chromosome 6p21.3. Circulation 1996;93:1493.

6. Houck KA, Leung DW, Rowland AM, et al. Dual regulation of vascular endothelial growth factor bioavailability by genetic and proteolytic mechanisms. J Biol Chem 1992;267: 26031.

7. Park JE, Keller GA, Ferrara N. The vascular endothelial growth factor (VEGF) isoforms: Differential deposition into the subepithelial extracellular matrix and bioactivity of extracellular matrix-bound VEGF. Mol Biol Cell 1993;4: 1317.

8. Hoeben A, Landuyt B, Highley MS, et al. Vascular endothelial growth factor and angiogenesis. Pharmacol Rev 2004; $56: 549$.

9. Küsters B, de Waal RM, Wesseling P, et al. Differential effects of vascular endothelial growth factor $A$ isoforms in a mouse brain metastasis model of human melanoma. Cancer Res 2003;63:5408.

10. Roodink I, van der LJ, Kusters B, et al. Development of the tumor vascular bed in response to hypoxia-induced VEGF-A differs from that in tumors with constitutive VEGF-A expression. Int J Cancer 2006;119:2054.

11. Fuh G, Wu P, Liang WC, et al. Structure-function studies of two synthetic antivascular endothelial growth factor Fabs and comparison with the avastin Fab. J Biol Chem 2006 281: 6625.

12. Presta LG, Chen $\mathrm{H}, \mathrm{O}^{\prime}$ Connor SJ, et al. Humanization of an antivascular endothelial growth factor monoclonal antibody for the therapy of solid tumors and other disorders. Cancer Res 1997;57:4593.

13. Ferrara N, Hillan KJ, Gerber HP, et al. Discovery and development of bevacizumab, an anti-VEGF antibody for treating cancer. Nat Rev Drug Discov 2004;3:391.

14. Tyagi P. Bevacizumab, when added to paclitaxel/carboplatin, prolongs survival in previously untreated patients with advanced non-small-cell lung cancer: preliminary results from the ECOG 4599 trial. Clin Lung Cancer 2005;6:276.

15. Jayson GC, Zweit J, Jackson A, et al. Molecular imaging and biological evaluation of HuMV833 anti-VEGF antibody: Implications for trial design of antiangiogenic antibodies. J Natl Cancer Inst 2002;94:1484.

16. Vermeulen PB, Colpaert C, Salgado R, et al. Liver metastases from colorectal adenocarcinomas grow in three patterns with different angiogenesis and desmoplasia. J Pathol 2001; 195:336.

17. Nagengast WB, de Vries EG, Hospers GA, et al. In Vivo VEGF Imaging with radiolabeled bevacizumab in a human ovarian tumor xenograft. J Nucl Med 2007;48:1313.

18. Stollman TH, Scheer MG, Leenders WP, et al. Specific imaging of VEGF-A expression with radiolabeled anti-VEGF monoclonal antibody. Int J Cancer 2008;122:2310.

19. Küsters B, Leenders WP, Wesseling $P$, et al. Vascular endothelial growth factor- $\mathrm{A}(165)$ induces progression of melanoma brain metastases without induction of sprouting angiogenesis. Cancer Res 2002;62:341.

20. Brouwers AH, van Eerd JE, Frielink C, et al. Optimization of radioimmunotherapy of renal cell carcinoma: Labeling of monoclonal antibody cG250 with ${ }^{131} \mathrm{I},{ }^{90} \mathrm{Y},{ }^{177} \mathrm{Lu}$, or ${ }^{186} \mathrm{Re}$. J Nucl Med 2004;45:327.

21. Span PN, Grebenchtchikov N, Geurts-Moespot J, et al. EORTC Receptor and Biomarker Study Group Report: A sandwich enzyme-linked immunosorbent assay for vascular endothelial growth factor in blood and tumor tissue extracts 10. Int J Biol Markers 2000;15:184.

22. Westphal JR, Van't Hullenaar R, Peek R, et al. Angiogenic balance in human melanoma: Expression of VEGF, bFGF, IL-8, PDGF, and angiostatin in relation to vascular density of xenografts in vivo. Int J Cancer 2000;86:768.

23. Stollman TH, Scheer MG, Leenders WP, et al. Specific imaging of VEGF-A expression with radiolabeled anti-VEGF monoclonal antibody. Int J Cancer 2008;122:2310.

24. Kusters B, Kats G, Roodink I, et al. Micronodular transformation as a novel mechanism of VEGF-A-induced metastasis. Oncogene 2007;26:5808. 